\title{
Colocación de implantes inmediatos post extracción en alvéolos de molares mandibulares con y sin injerto óseo. Presentación de casos clínicos.
} Immediate implant placement into fresh extraction mandibular molar sockets with or without bone graft. Report of clinical cases.

\section{Diego Robalino Torres ${ }^{1}$ Fernando Sandoval Portilla ${ }^{2}$ Fernando Sandoval Vernimmen ${ }^{3}$}

1. Universidad San Francisco de Quito, Colegio de Ciencias de la Salud, Escuela de Odontología, Clínica Odontológica, Campus Cumbayá, oficina C0 106, casilla postal 17-1200841. Quito-Ecuador. Correo electrónico: alejodr87@hotmail.com

Universidad San Francisco de Quito, Colegio de Ciencias de la Salud, Escuela de Odontología, Clínica Odontológica, Campus Cumbayá, oficina C0 106, casilla postal 17-1200841. Quito-Ecuador. Correo electrónico: fjose28@hotmail.com

3. Universidad San Francisco de Quito, Colegio de Ciencias de la Salud, Escuela de Odontología, Clínica Odontológica, Campus Cumbayá, oficina C0 106, casilla postal 17-1200-841. Quito-Ecuador. Correo electrónico: fsandoval@usfq.edu.ec

Editado por / Edited by: Johanna Monar

Recibido / Received: 30-08-2019

Aceptado / Accepted: 3-02-2020 


\section{Resumen}

El objetivo la presentación de esta serie de casos fue evaluar las diferencias y las reacciones de los tejidos peri implantares en implantes inmediatos postextracción en alvéolos de molares mandibulares infectados o no infectados, con o sin colocación de injerto óseo. Se colocaron cuatro implantes inmediatos postextracción de molares mandibulares con o sin infección en cuatro pacientes, fueron divididos en 2 grupos ( $n=2$ ), uno sin injerto óseo y otro con injerto óseo. Se realizó controles clínicos y radiográficos a los 8 días y a los 4 meses de colocado el implante. Ambos grupos presentaron oseointegración de los implantes con una taza de supervivencia del $100 \%$. No se observó mayor diferencia a nivel de la cresta ósea en sentido vertical ni horizontal en las evaluaciones radiográficas. En la presentación de los 4 casos clínicos, se tuvo éxito en ambos grupos con un seguimiento mínimo de 4 meses.

Palabras clave: Implante inmediato, injerto óseo, molar mandibular, alvéolo fresco postextracción, alvéolo infectado, cicatrización ósea.

\section{Abstract}

The aim of this series of cases was to evaluate the differences and reactions of tissues around immediate implants placed in infected or noninfected sockets in mandibular molars, with or without bone graft placement. Four immediate implants were placed in infected or non-infected mandibular molar sockets in four patients. The patients were divided into 2 groups $(n=2)$. In Group 1 a bone graft was placed and in Group 2 no bone graft was placed. Clinical and radiographic evaluations were performed 8 days and 4 months after implant placement in all patients. At 4 months follow-up, both groups showed favorable bone-implant integration, and the survival rate of both groups was $100 \%$. No greater difference was observed between the 2 groups to the radiographic evaluation at 8 days and 4 months related to changes in the level of the bone crest in the vertical and horizontal direction. No major difference was observed at the level of the bone crest vertically or horizontally in the radiographic evaluations. In the presentation of the 4 clinical cases, it was successful in both groups with a minimum follow-up of 4 months.

Keywords: Immediate implant, bone graft, mandibular molar, fresh extraction socket, infected socket, bone healing. 


\section{Introducción}

La pérdida de los dientes se puede producir por múltiples causas, entre ellas se encuentran: enfermedad, trauma dental o ausencia congénita. La colocación de implantes dentales se estableció como una opción de tratamiento para reemplazar dientes perdidos, permitiendo la restauración de función masticatoria, fonación, y estética (1)(2)

Tradicionalmente, antes de colocar los implantes dentales, se retiran los dientes comprometidos y se deja cicatrizar los alvéolos de extracción durante varios meses. Sin embargo, la reabsorción del reborde alveolar después de extracción dental puede reducir considerablemente el volumen de hueso residual y comprometer el posicionamiento favorable de los implantes necesarios para la restauración óptima (2)(3). Se describe como implante inmediato al proceso mediante el cual se coloca un implante en el mismo acto quirúrgico en que se realiza la extracción del diente a ser sustituido (4).

La colocación inmediata de los implantes dentales en alvéolos de molares también se ha presentado como una alternativa exitosa al protocolo diferido. Las tasas de supervivencia para los implantes en molares colocados inmediatamente son similares a la de los implantes colocados en la extracción de molares de forma convencional (3)(4).

En casos clínicos que requieren de extracción dental y colocación inmediata de implantes se ha observado presencia de patología periapical y/o periodontal, sin embargo, varios autores sugieren que la colocación inmediata de implantes en un sitio infectado no está contraindicada, como en sitios con patología que se pensaba que comprometían la oseointegración (5)(6)(7)(8).

Actualmente se realiza la extracción y la colocación del implante en un solo tiempo quirúrgico, lo cual brinda beneficios con respecto a la menor cantidad de intervenciones quirúrgicas, disminución de tiempo de cicatrización y un adecuado mantenimiento de la arquitectura alveolar (9)(10)(11)(12).

\section{Presentación de casos}

En el presente estudio de tipo clínico comparativo prospectivo, se seleccionaron 4 pacientes que acudieron al servicio de Cirugía Oral y Maxilofacial de la clínica Odontológica de la Universidad San Francisco de Quito - Ecuador en el periodo de enero del 2015 a diciembre del 2017, cuyos molares en el área mandibular tuvieron indicación de extracción dental. Se dividió a los pacientes seleccionados en dos grupos, de los cuales se asignó un grupo control (2 pacientes: casos 1 y 2) con implantes inmediatos postextracción en el área molar mandibular sin injerto óseo y un grupo de estudio ( 2 pacientes: casos 3 y 4 ) con implantes inmediatos postextracción en el área molar mandibular con injerto óseo. 


\section{Selección de pacientes}

Se incluyeron en este estudio pacientes con:

- Mayoría de edad, sistémicamente saludables.

- Indicación para extracción de un molar mandibular con o sin lesión periapical.

- Oclusión funcional y estable.

- Presencia de hueso vestibular y lingual intactos.

- Presencia de margen gingival sano.

- Longitud ósea mínima de 3 mm desde la porción apical del alvéolo hasta la cortical superior del conducto dentario inferior.

- Capacidad de proporcionar un consentimiento informado.

- Higiene oral aceptable.

- Ausencia de exudado o supuración.

- Presencia o ausencia de patología periapical o periodontal crónica no supurativa.

Se excluyó de este estudio a pacientes cuyos molares mandibulares presenten:

- Pérdida parcial o total de la pared ósea bucal.

- Procesos infecciosos periapicales y/o periodontales agudos con presencia de supuración.

- Pacientes con enfermedades no controladas o cualquier condición que pueda causar problemas con la oseointegración.

- Pacientes con hábitos parafuncionales como bruxismo.

- Pacientes con higiene oral pobre.

\section{Protocolo quirúrgico}

Previo a la intervención quirúrgica, los 4 pacientes recibieron 625 mg de Amoxicilina más ácido clavulánico 8 horas antes del procedimiento. Bajo protocolos de asepsia y antisepsia en cada paciente se realizó infiltración anestésica de Lidocaína 2\% más epinefrina 1:80:000 con la técnica de anestesia troncular mandibular debido a la extracción del lado a ser tratado, posteriormente se llevó a cabo una incisión pequeña con hoja de bisturí número 15 en el área bucal a cada lado del al- 
véolo. Se levantó un colgajo bucal mucoperióstico que respetó la papila dental de las piezas vecinas, después se realizó la extracción de la pieza dental con técnicas atraumáticas mínimamente invasivas. La odontosección de las raíces se llevó a cabo con una fresa de fisura n.- 701. Luego de la extracción, se realizó curetaje de la cavidad alveolar con curetas quirúrgicas de Lucas y solución salina estéril en todos los casos. (Fig. 1).
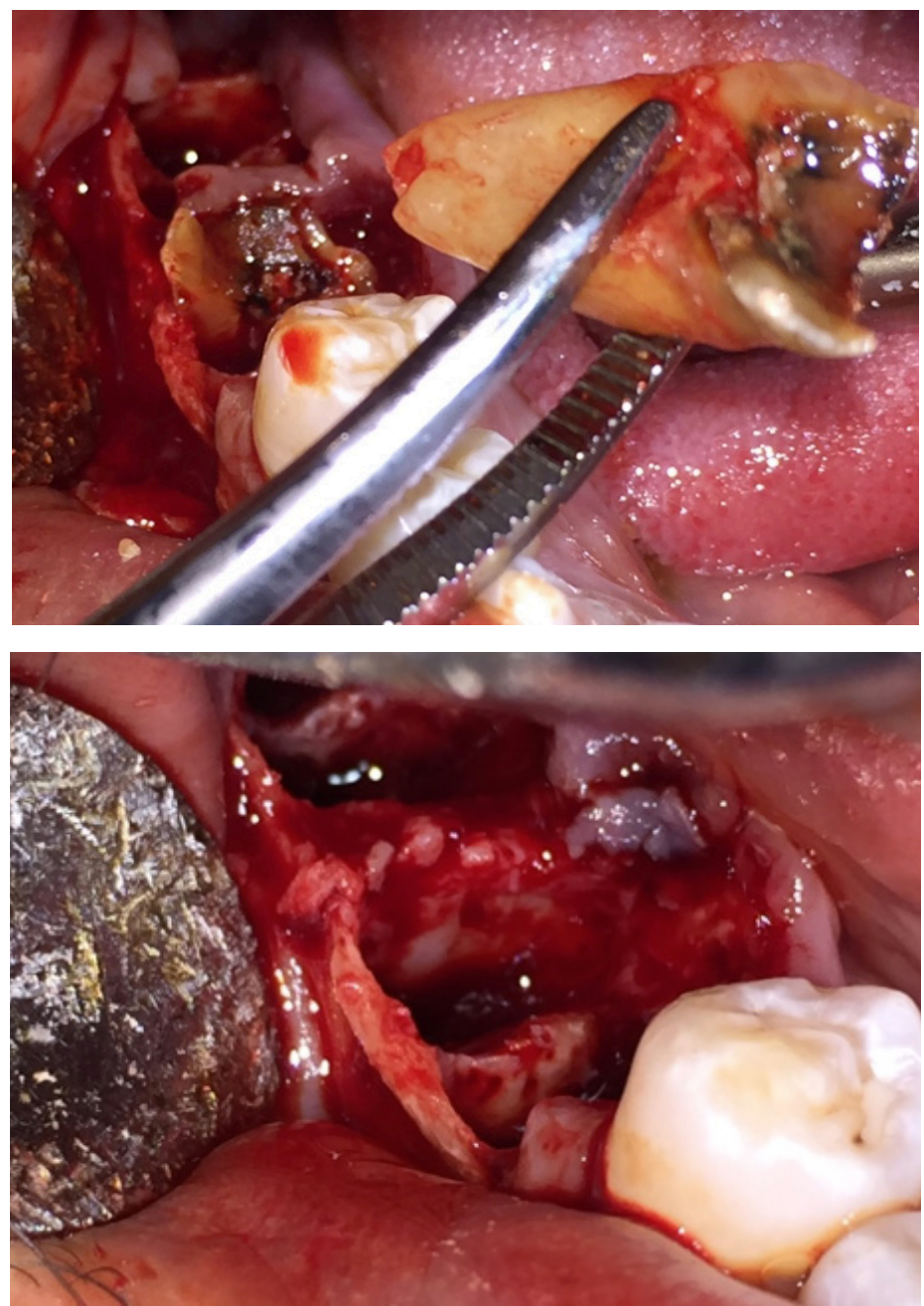

Figura 1. Caso 1. Extracción de implante mínimamente invasiva con odontosección y curetaje de la lesión. 
Se inició la perforación del tejido óseo con las fresas quirúrgicas correspondientes al kit quirúrgico de implantes del sistema utilizado. Los implantes fueron colocados en un hueso apical sano mínimo de $3 \mathrm{~mm}$ con un torque mandatorio de inserción mínimo de $35 \mathrm{Ncm}$. (Fig. 2).

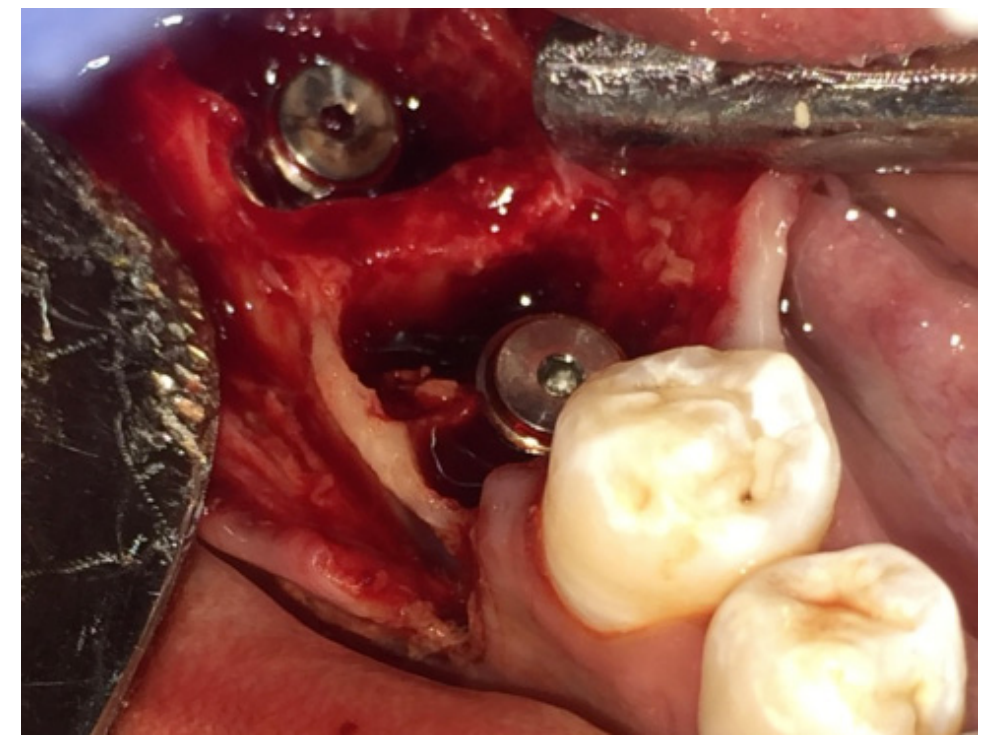

\section{Figura 2. Caso 1. Colocación de implantes dentales postextracción en sector de piezas 46-47 sin colocación de injerto óseo.}

Luego de colocado el implante, en el colgajo bucal desplegado se realizó la maniobra de Rehrmann hasta avanzar el colgajo sin tensión al margen gingival lingual. El colgajo bucal fue fijado sin tensión mediante puntos de sutura simple con material no absorbible seda 4-0.

En el grupo donde se utilizó injerto óseo (casos 3 y 4), previo al cierre del colgajo mediante puntos de sutura, se colocó las partículas del injerto óseo hidratadas entre en el espacio del alvéolo y el implante.

En el caso 1 se realizó la extracción de la pieza dental 46, 47, 48 y se colocaron dos implantes inmediatos, se tomó en cuenta un solo implante (pieza 46) del sistema I-fix de $13 \mathrm{~mm}$ de longitud y 5,0 mm diámetro, cuyo torque de inserción fue de 40 Ncm. (Fig. 1,2). 
En el caso 2 se realizó la extracción de la pieza dental 47 y se colocó un implante inmediato del sistema l-fix, de $13 \mathrm{~mm}$ de longitud y 5,0 mm de diámetro, cuyo torque de inserción fue de $43 \mathrm{Ncm}$ (Fig. 3).
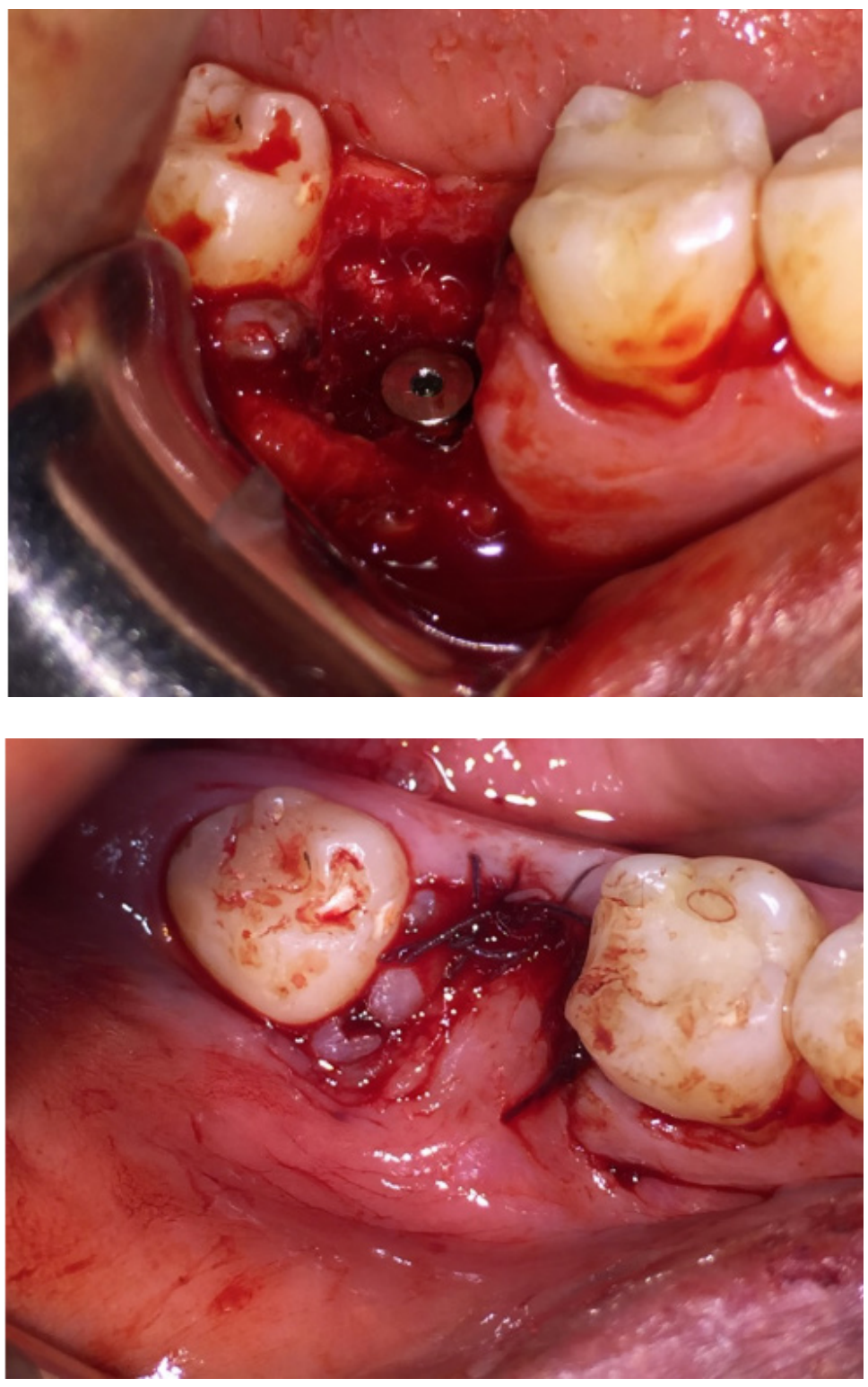

Figura 3. Caso 2. Colocación de implante inmediato postextracción sin colocación de injerto óseo en sector de pieza 47. 
En el caso 3 se realizó la extracción de la pieza dental 36, se colocó un implante inmediato del sistema TSV de $13 \mathrm{~mm}$ de longitud y 4,7mm de diámetro, cuyo torque de inserción fue de $40 \mathrm{Ncm}$. Se colocó un injerto óseo particulado entre el implante y el alvéolo (Fig. 4).
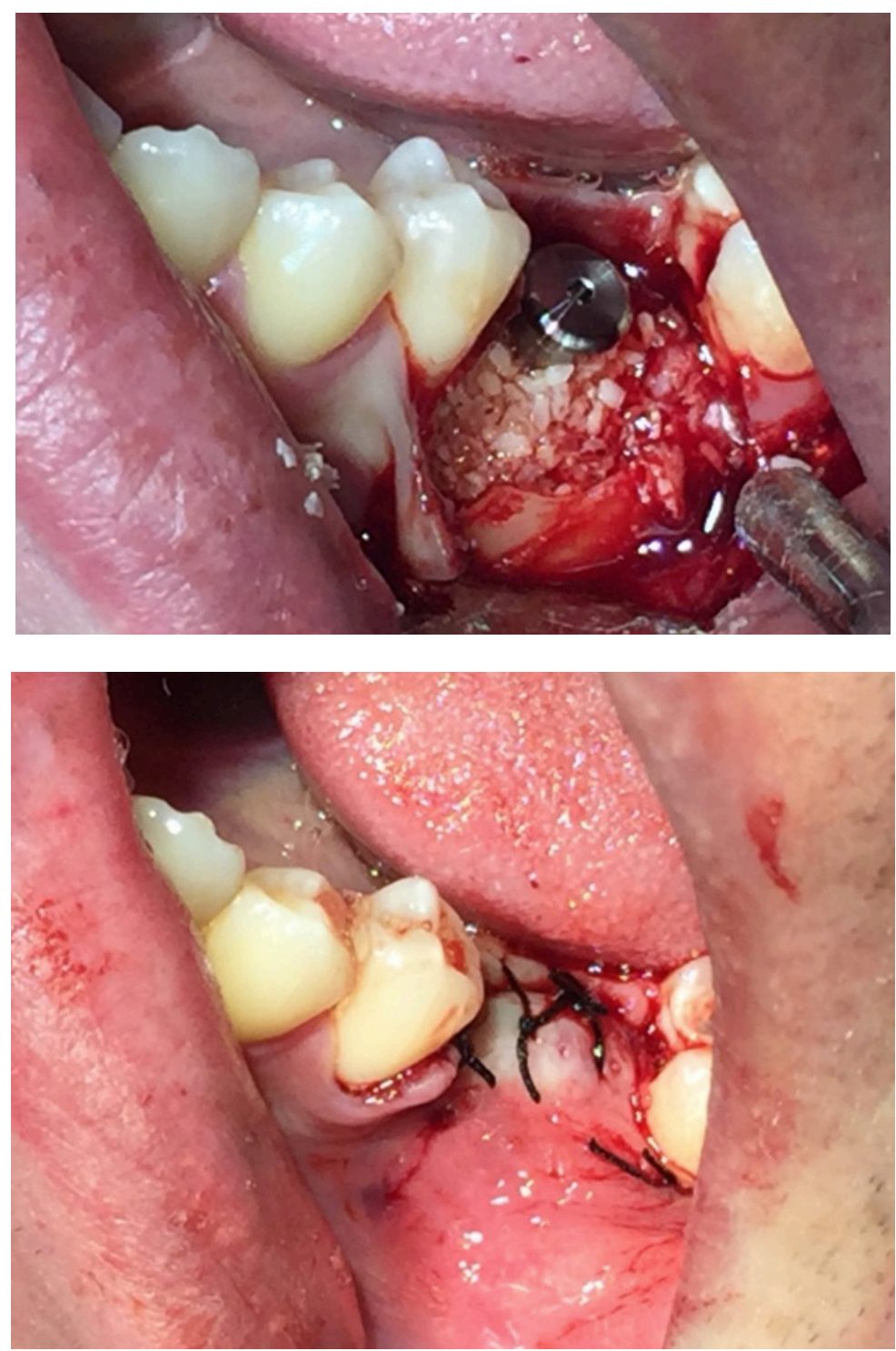

Figura 4. Caso 3. Colocación de implante inmediato postextracción con colocación de injerto óseo en sector de pieza 36. 
En el caso 4 se realizó la extracción de la pieza dental 46 y se colocó un implante inmediato postextracción del sistema l-fix de $11,5 \mathrm{~mm}$ de longitud y $5,0 \mathrm{~mm}$ de diámetro, cuyo torque de inserción fue de $45 \mathrm{Ncm}$. Se colocó un injerto óseo particulado entre el implante y el alvéolo (Fig. 5).
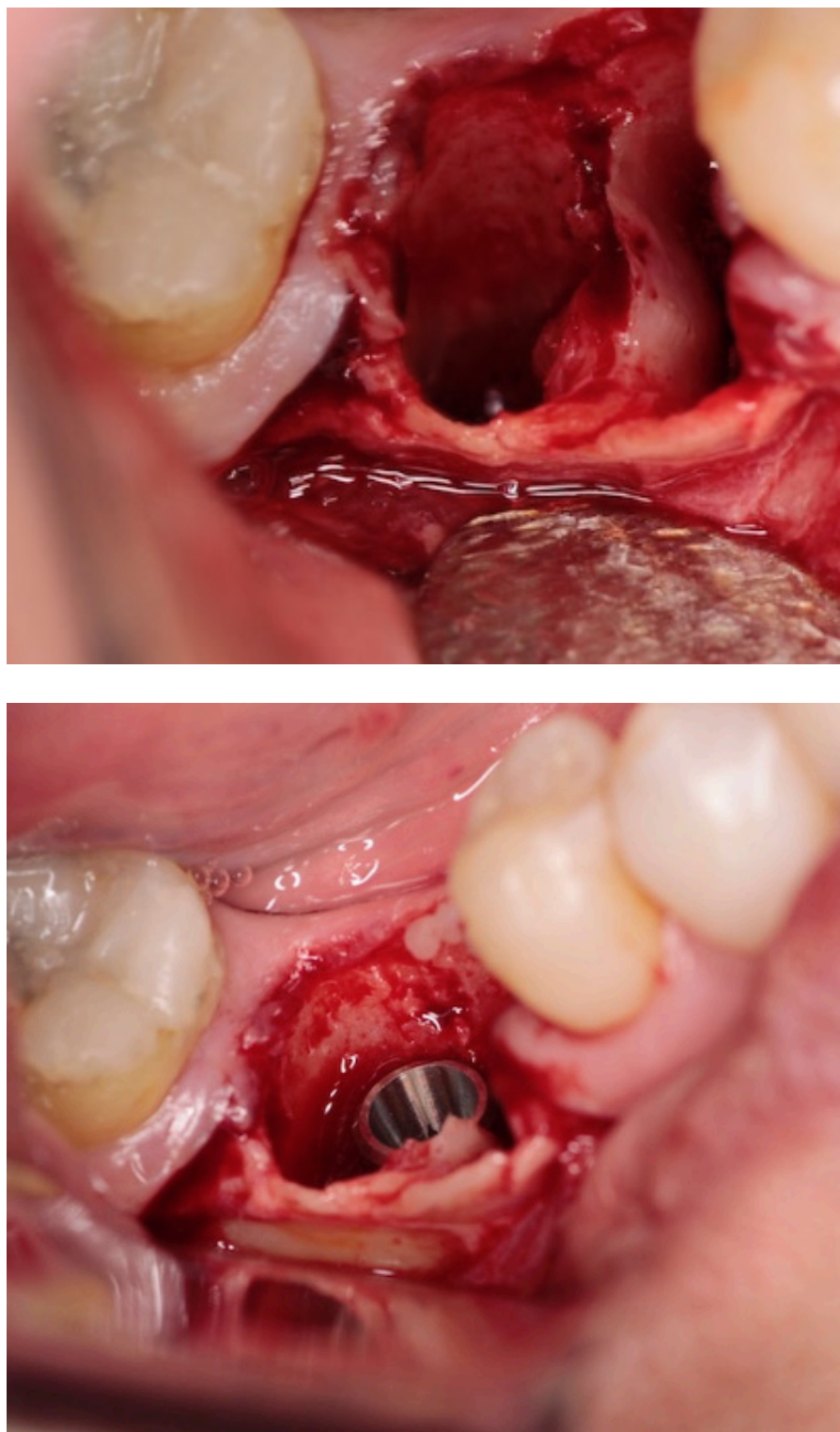

Figura 5. Caso 4. Colocación de implante inmediato postextracción en sector de pieza 46 con colocación de injerto óseo. 
En los casos 1, 2 y 3 previo a la colocación del implante se realizó la remoción de la lesión apical, curetaje, limpieza e irrigación del alvéolo.

Los puntos de sutura fueron retiradas a los 8 días después de la cirugía. Todos los pacientes recibieron las mismas indicaciones postoperatorias.

\section{Selección del implante y remanente óseo}

Los implantes utilizados en este estudio fueron del sistema I-FIX, (Titanium Fix, São José dos Campos, Brasil) y del sistema Tapered Screw-Vent (TSVTM Implant System, Zimmer Biomet Dental, Palm Beach, Florida, U.S.A). Se usaron implantes cilindro cónicos y cónicos de 4.5 y $5 \mathrm{~mm}$ de diámetro con una longitud variable de acuerdo al caso entre 11.5 a $13 \mathrm{~mm}$. En todos los casos se requirió una longitud mesiodistal y bucolingual mínima de $7 \mathrm{~mm}$ y una altura mínima de $11 \mathrm{~mm}$ desde el límite cervical de la pared vestibular hasta el conducto mandibular para la colocación de implantes con un diámetro mínimo de 4,5 mm y una longitud mínima de 10 $\mathrm{mm}$. También fue necesaria una longitud ósea mínima de $3 \mathrm{~mm}$ desde la porción apical del alvéolo hasta el canal mandibular.

\section{Selección del injerto óseo}

Se utilizó como injerto óseo una matriz ósea desmineralizada y liofilizada compuesta por partículas de 300 a 600 micras de hueso cortico-esponjoso de origen de fémur porcino (Biomod, 3 Biomat, Bogotá D.C., Cundinamarca, Colombia) en el espacio entre el alvéolo y el implante cubriéndolo en su totalidad, previamente se realizó la hidratación del injerto óseo con solución salina.

\section{Evaluación clínica y radiográfica}

En las citas de control se realizó la valoración clínica por un solo examinador, calibrado y estandarizado. La medición radiográfica del tejido óseo después de realizada la extracción y colocado el implante se realizó mediante el equipo de radiografía panorámica digital (Trophypan, Trophy, Paris, Isla de Francia, Francia) con el software de radiografía panorámica digital (CDR-DICOM para Windows versión 4.5.0, Schick technologies Inc., New York, EE. UU.).

Las mediciones radiográficas en los pacientes de este estudio se realizaron en 3 controles: antes de la colocación del implante para el diagnóstico inicial, 8 días después de la colocación del implante y a los 4 meses después de colocado el implante.

\section{Resultados}

Todos los implantes presentaron estabilidad primaria en el momento de su inserción, no se hallaron signos de infección, dehiscencia, inflamación o sangrado en ningún caso. A la valoración de las condiciones los tejidos blandos peri implantares no se observó mucositis, por lo tanto, las unidades peri implantares no mostraron ningún grado de inflamación. En los controles radiográficos en sentido horizontal 
y vertical por mesial y distal, a los 8 días y los 4 meses, no se observó pérdida del implante, ausencia de oseointegración o presencia de sombras radiopacas alrededor del implante en ningún caso (Fig 6-9).

Caso 1. Control vertical mesial
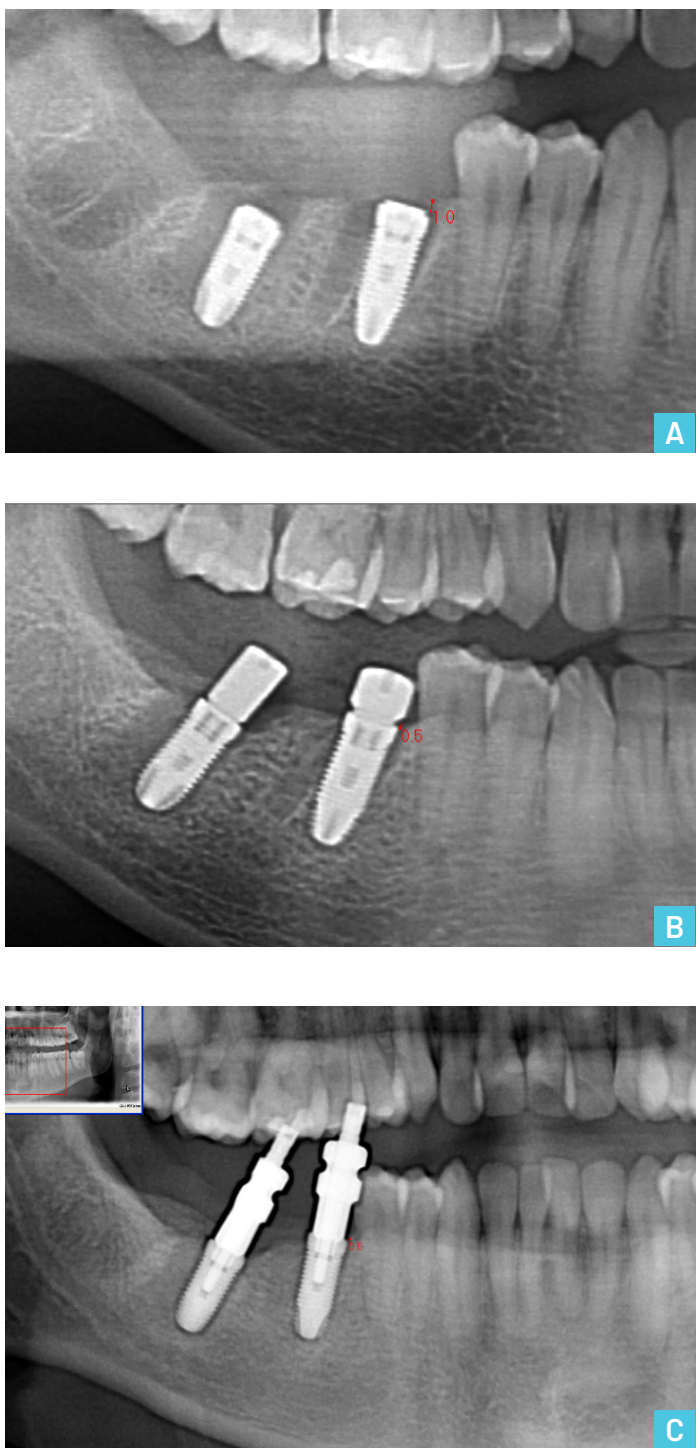

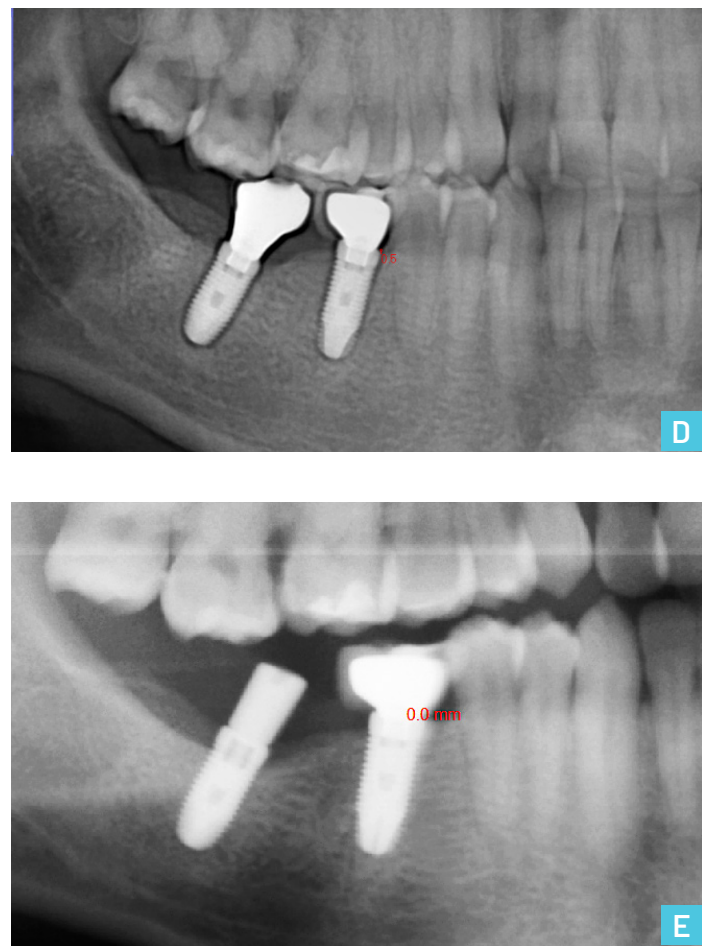

Figura 6. Caso 1. Control radiográfico vertical $(\mathrm{mm})$ mesial del implante 46 . A. Control a los 8 días. B. Control a los 4 meses. C. Control a los 8 meses D. Control a los 10 meses. E. Control a los 18 meses.

\section{Caso 2. Control vertical distal}

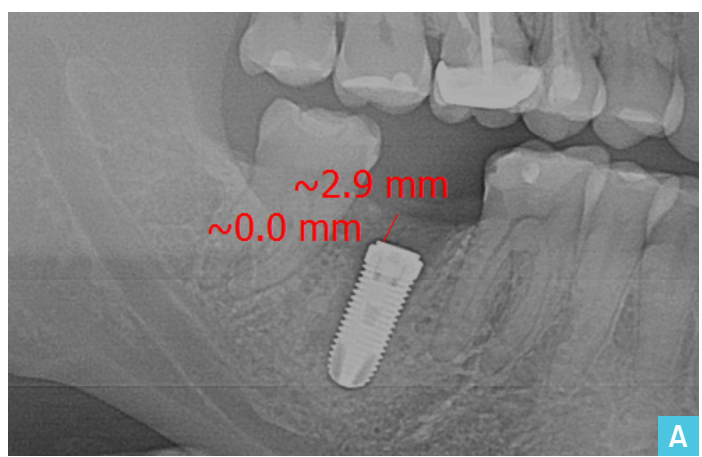




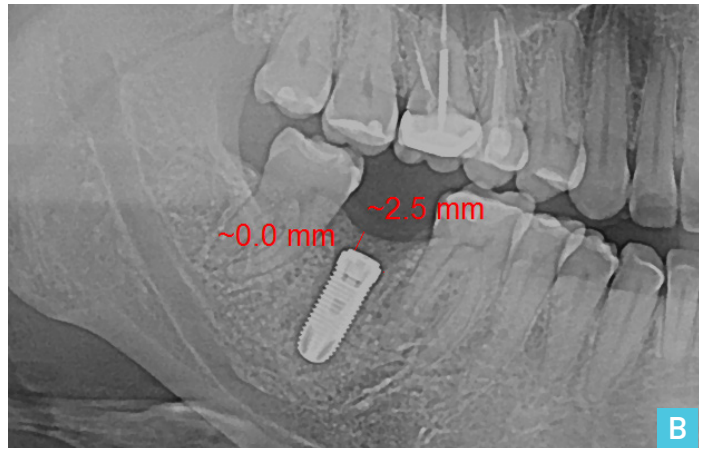

Figura 7. Caso 2. Control radiográfico vertical $(\mathrm{mm})$ distal del implante 47. A. Control a los 8 días. B. Control a los 4 meses.

Caso 3. Control horizontal mesial
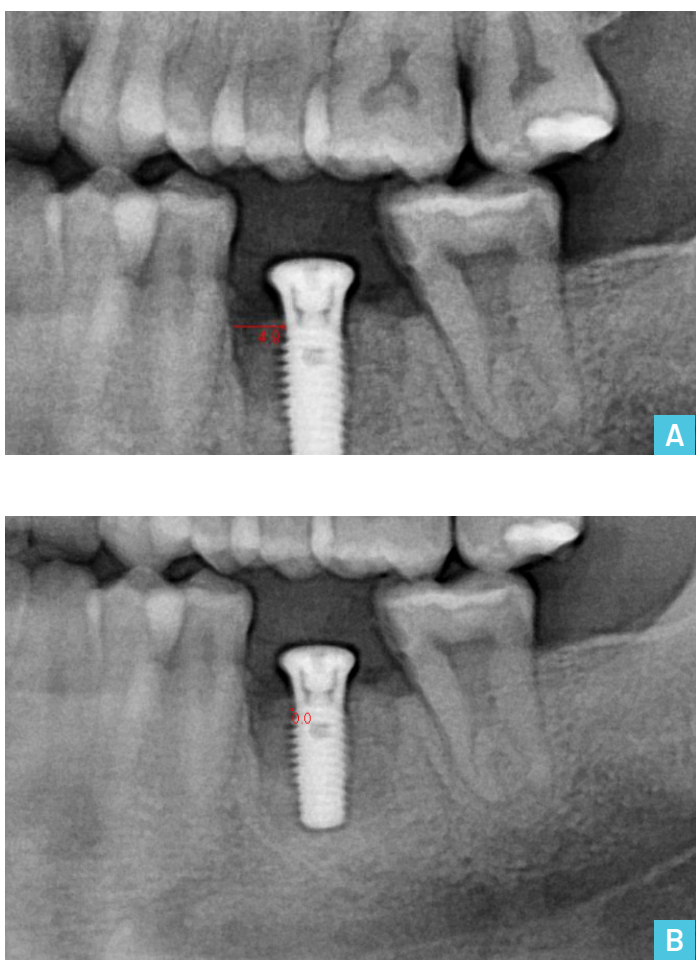


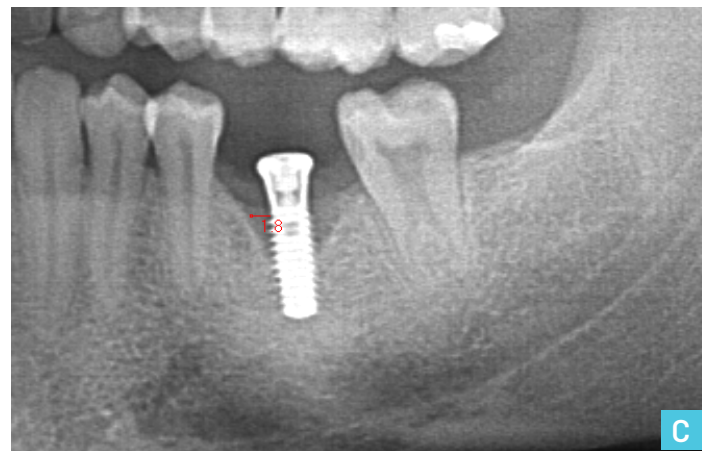

Figura 8. Caso 3. Control radiográfico horizontal $(\mathrm{mm})$ mesial del implante 36.

A. Control a los 8 días. B. Control a los 4 meses. C. Control a los 24 meses.

\section{Caso 4. Control horizontal distal}
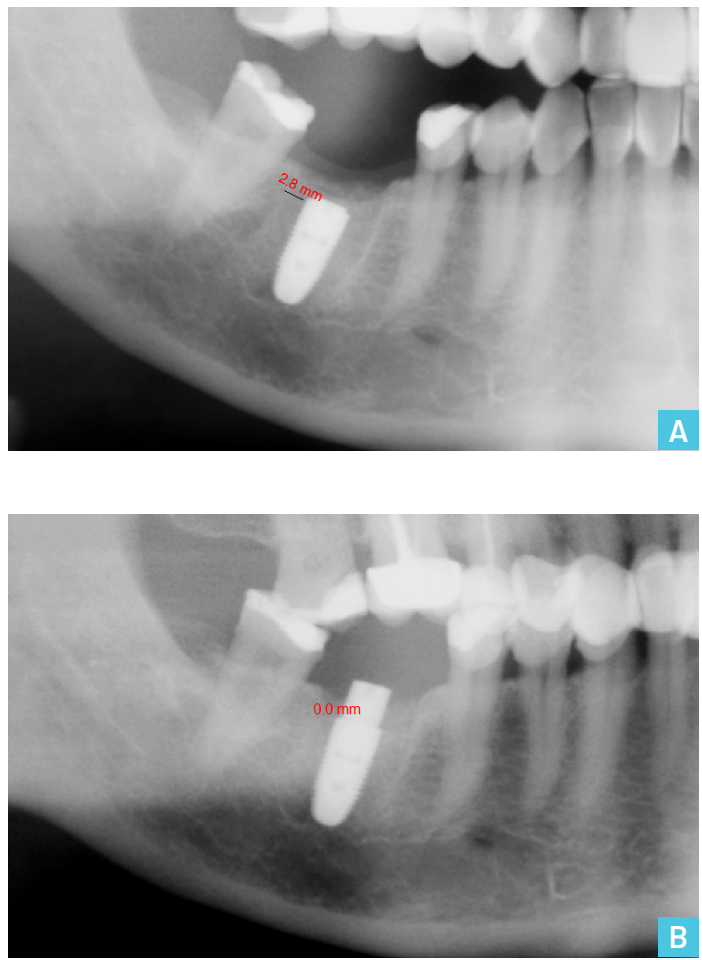

Figura 9. Caso 4. Control radiográfico horizontal $(\mathrm{mm})$ distal del implante 46. A. Control a los 8 días. B. Control a los 4 meses. 
La variación observada de pérdida ósea a los 4 meses en los promedios de los casos con injerto y sin injerto fueron en vertical mesial de 0 y 0,3 $\mathrm{mm}$ y en distal de 0 a 0,6 mm respectivamente. En sentido horizontal la variación de los casos con injerto y sin injertos fueron en mesial de 0 a $0,5 \mathrm{~mm}$ y en distal de 0 a $0,3 \mathrm{~mm}$.

En el grupo de casos donde no se colocó injerto óseo, se observó a los 4 meses en sentido vertical pérdida ósea mesial y distal similar y se observó en sentido horizontal formación ósea mesial y distal similar. No hubo diferencia entre los dos casos sin colocación de injerto óseo.

En el grupo de casos donde se colocó injerto óseo entre el implante y el alvéolo, se observó a los 4 meses en sentido vertical menor pérdida ósea en comparación a los casos donde no se colocó injerto óseo y en sentido horizontal se observó en ambos casos con injerto una formación ósea completa a los 4 meses.

En el caso 3, donde se colocó injerto óseo y no se realizó rehabilitación del implante, a los 24 meses se observó una mayor reabsorción ósea vertical y horizontal.

No se encontró mayor diferencia en los cambios de la cresta ósea en sentido vertical, ni se observó cambios en la formación ósea en sentido horizontal entre los casos donde se usó injerto óseo y en aquellos casos donde no se usó injerto óseo como material de regeneración.

\section{Discusión}

La colocación de implantes inmediatamente después de la extracción del diente ha demostrado ser una modalidad de tratamiento exitosa en ensayos clínicos y modelos experimentales. La disminución de los procedimientos quirúrgicos y duración del tratamiento restaurador presenta beneficios estéticos y funcionales (13), (11). Al igual que otros estudios, creemos que con una evaluación exhaustiva de cada caso, la extracción de los dientes residuales y la colocación del implantes en un solo procedimiento quirúrgico es una modalidad de tratamiento segura y predecible (14).

Un estudio de Bersani y col. (10), realizó la colocación de 23 implantes inmediatos postextracción en un periodo de 6 años con un seguimiento mínimo de 4 meses. Como resultado todos los implantes tuvieron estabilidad, no presentaron signos de infección, inflamación, sangrado o pérdida del implante. En comparación con nuestro estudio de 4 implantes inmediatos postextracción con un periodo de seguimiento similar, los resultados muestran concordancia con dicho estudio en cuanto a la estabilidad de los implantes. No se registraron pérdidas o aparición de signos de inflamación, sangrado e infección. Esto puede ser explicado por la presencia de paredes óseas alveolares intactas, suficiente remanente óseo apical con relación al conducto mandibular, torque mínimo de inserción de 35 Ncm y colocación de implantes con diámetro mínimo de 4,5 mm con longitud mínima de $10 \mathrm{~mm}$ en ambos estudios. 
Un estudio de cohorte retrospectivo (15), donde se colocaron 59 implantes inmediatos postextracción en el sector posterior, evaluó la regeneración ósea y de los tejidos blandos en alvéolos con defectos de dehiscencia en la pared bucal a 2 niveles de altura distintos y alvéolos intactos sin defectos. En todos los casos se colocaron materiales de regeneración ósea y no se levantaron colgajos, a los 6 meses los resultados indicaron que el volumen de regeneración ósea en sentido horizontal y vertical, la disminución del hueso crestal en relación con la plataforma del implante y la regeneración de tejidos blandos, fueron similares en todos los grupos sin una diferencia significativa. Con similares resultados en nuestro estudio, presumimos que la cicatrización de los tejidos en alvéolos postextracción depende del mantenimiento del periostio, cuya capa interna contiene células madre mesenquimales multipotentes y células osteoprogenitoras que contribuyen al crecimiento normal del hueso, la cicatrización y regeneración ósea(16).

En una revisión sistemática y meta-análisis por Mello y col. (9), se compararon la supervivencia y los cambios peri implantares asociados de dos grupos: un grupo de implantes inmediatos en alvéolos frescos y otro grupo de implantes colocados en alvéolos cicatrizados. Los autores analizaron 30 estudios donde se colocaron 3049 implantes, determinando una tasa de supervivencia del $98.38 \%$ en implantes convencionales y de $95.21 \%$ en implantes inmediatos. Se analizó también la pérdida ósea marginal, la estabilidad y la profundidad de sondaje y se observó que no hubo diferencia significativa entre los 2 grupos. A pesar de las limitaciones de nuestro estudio, los resultados en cuanto a supervivencia, estabilidad, pérdida ósea marginal están en concordancia con lo descrito por Mello y col. (9). Es posible atribuir estos resultados a que el proceso de remodelación de la cresta es completado en promedio a las 16 semanas de la extracción, tiempo considerado para la colocación convencional de implantes y en el cual los implantes postextracción pueden garantizar alta estabilidad. En nuestro estudio, el 100\% de los implantes presentó estabilidad un periodo de seguimiento mínimo de 4 meses y máximo de 24 meses. Un estudio retrospectivo (17) de 95 implantes inmediatos presentó resultados similares con una supervivencia del $95 \%$ en un periodo de 4 años en comparación con el estudio de Mello y col.

Con respecto al espacio entre la superficie interna del alvéolo y la superficie del implante, en un estudio (12) se analizó 35 implantes inmediatos en molares, donde luego de colocado el implante se rellenó el espacio con una mezcla de hueso autólogo recolectado del fresado inicial y hueso alogénico, observando una adecuada estabilidad y preservación alveolar. En el estudio de Bersani y col. (10), se colocaron 23 implantes inmediatos en el área molar sin necesidad de la colocación de materiales de relleno o membranas y no se encontraron signos de pérdida ósea o de falta de integración de los implantes. En comparación con nuestro estudio, en el cual realizamos la colocación de injerto óseo en dos casos, y en 2 casos no colocamos injerto óseo, no observamos diferencia entre los dos grupos con respecto a formación y pérdida ósea, esto puede ser explicado debido a la preservación de la pared bucal intacta y de los tejidos blandos que proveen vascularidad al hueso alveolar. Un estudio experimental realizado en perros (18), examinó los cambios del hueso alveolar y del tejido blando en distancias diferentes entre el implante y el hueso alveolar que fueron de 1, 2 y $3 \mathrm{~mm}$ respectivamente. A través de análisis 
morfométricos y de tomografía microcomputada, determinaron que el grupo con una distancia de $3 \mathrm{~mm}$ tenía mayor volumen de formación ósea y de tejido blando con una menor reabsorción ósea en un periodo de control de 4 meses. Los autores observaron que cuanto más ancho fue el espacio bucal hubo mayor formación de tejido óseo y blando.

De acuerdo a Scwartz-Arad \& Chaushu (17), el cierre del colgajo es un importante componente en la colocación de implantes inmediatos. Furgazzotto (19), en su estudio de serie de casos, donde se colocaron 83 implantes inmediatos en molares maxilares con injertos óseos y membranas bioabsorbibles, cubrió en todos los casos el implante y los materiales de regeneración con un colgajo bucal mucoperióstico proporcionando un cierre primario de los tejidos blandos, el mismo que se mantuvo 6 meses hasta el momento de la cirugía de cicatrización. En otras publicaciones (10), (20) y (21) en los implantes colocados no se realizó un cierre primario de los tejidos debido a que fueron sometidos a carga funcional luego de su colocación, mostrando estabilidad de los mismos. En nuestro estudio, los implantes no fueron sometidos a carga inmediata por lo que realizamos un cierre primario en el área molar en todos los casos. No se hallaron diferencias con respecto a estabilidad del implante, presencia de complicaciones como dehiscencia de los tejidos con relación a los estudios que no realizaron el cierre primario. Es probable que el resultado observado se deba a que la protección del coágulo inicial luego de la colocación de implantes contra elementos que impidan la cicatrización sea esencial para mantener los tejidos peri implantares, pudiéndose conseguir a través de colgajos o de forma protésica, de modo que entre el alvéolo y el implante se inicien los procesos biológicos que promueven regeneración normal de dichos tejidos.

La colocación de implantes inmediatos en sitios infectados ha sido un tema controversial. Un meta-análisis (6), donde se comparó la colocación de implantes inmediatos postextracción en alvéolos infectados y no infectados, sugiere que la colocación inmediata en sitios infectados incrementa el riesgo de falla del implante. En un estudio (8) se evaluaron 168 implantes inmediatos colocados en alvéolos con y sin infección distribuidos en 3 grupos: alvéolos no infectado, alvéolos con infección aguda y alvéolos con infección crónica. Según este estudio, las tazas de supervivencia en el periodo de un año fueron de $98.8 \%, 100 \%$ y $94 \%$ respectivamente donde no se hallaron diferencias significativas. En nuestro estudio, se observó radiográficamente presencia de lesiones apicales en 3 casos. En dichos casos luego de la extracción, realizamos el curetaje, remoción completa de la lesión, irrigación y limpieza del alvéolo con solución salina en el alvéolo antes de la colocación del implante. Al igual que otros estudios (2)(7),(8),(22) los resultados fueron satisfactorios, no hallamos diferencia en la supervivencia entre la colocación de implantes en sitios con o sin infección. Esto puede ser explicado debido a que la técnica quirúrgica incluye procedimientos como la remoción de la lesión, desbridamiento, limpieza de la cavidad alveolar, prescripción de antibióticos sistémicos, uso de enjuague bucal con soluciones antimicrobianas y aplicación de técnicas de regeneración ósea y tisular guiada, que disminuyen el riesgo de fracaso en los implantes. Una reciente revisión sistemática y metaanálisis (5) donde se incluyeron 9 estudios publicados entre el año 2010 al 2017 de implantes inmediatos postextracción en alvéolos infectados en zona estética, evaluó un total de 1735 
participantes, de los cuales 758 pertenecían al grupo infectado y 977 al grupo no infectado. Se observaron tazas similares de supervivencia $97.6 \%$ en sitios infectados versus $98.4 \%$ en sitios sin infección sin diferencias significativas.

Uno de los implantes de este estudio (caso 3 con injerto óseo), no fue rehabilitado en el periodo recomendado por decisión del paciente, este fue el único que presentó características de reabsorción ósea mayor en un periodo de control de 24 meses. Creemos que la reabsorción en este caso se debe a la falta de estimulación funcional por medio de la prótesis no permitió una adecuada remodelación del tejido óseo alrededor del implante. En un estudio prospectivo (23), donde se realizó la evaluación radiográfica de 596 implantes en 192 pacientes, observaron una remodelación clínicamente significativa del hueso marginal durante los primeros 6 meses después de la colocación del implante. Posteriormente se produjo una pérdida ósea de 0,22 a 0,42 mm entre el momento de la colocación de la prótesis y la poscarga de 1 año. Luego la pérdida ósea observada alrededor de los implantes hasta 5 años después de la carga fue mínima.

Estudios futuros con un periodo de observación más largo y un tamaño de muestra mayor, serán necesarios para elucidar y explicar en profundidad los mecanismos relacionados al proceso de formación ósea en estos casos.

\section{Conclusiones}

- Con las limitaciones del presente estudio, tales como el pequeño numero de participantes, podemos concluir que la colocación de implantes inmediatos postextracción en el área molar mandibular con el uso de injertos óseos en pacientes, no presenta diferencias con respecto al no uso de injertos, por lo tanto, el uso de injertos óseos no es indispensable y podría ser omitido.

- La colocación de los implantes inmediatos postextracción en molares mandibulares con o sin injerto óseos, con o sin levantamiento del colgajo, con o sin presencia de procesos infecciosos periapicales o periodontales crónicos, puede ser considerado como un tratamiento predecible y con alta supervivencia, en situaciones clínicas donde las condiciones óseas sean óptimas, con altura y espesor suficientes. 


\section{Referencias bibliográficas}

1. Al-sabbagh M, Kutkut A. Immediate Implant Placement Surgical Techniques for Prevention and Management of Complications. Dent Clin NA. Elsevier Inc; 2015;59(1):73-95.

2. Del Fabbro M, Boggian C, Taschieri S. Immediate implant placement into fresh extraction sites with chronic periapical pathologic features combined with plasma rich in growth factors: preliminary results of single-cohort study. J Oral Maxillofac Surg. Elsevier Inc.; 2009;67(11):2476-84.

3. Smith RB, Tarnow DDSDP. Classification of Molar Extraction Sites for Immediate Dental Implant Placement : Technical Note. J Oral Maxillofac Implant. 2013;28:911-6.

4. Peñarrocha M, Uribe R, Balager J. Implantes inmediatos a la exodoncia. Situación actual. Med Oral Oral. 2004;9(1):234-42.

5. Chen H, Zhang G, Weigl P, Gu X. Immediate placement of dental implants into infected versus noninfected sites in the esthetic zone: A systematic review and meta-analysis. $J$ Prosthet Dent. Editorial Council for the Journal of Prosthetic Dentistry; 2018;1-10.

6. Zhao D, Wu Y, Chun X, Zhang F. Immediate dental implant placement into infected vs. non-infected sockets : a meta-analysis. Clin Oral Implants Res. 2016;27:1290-6.

7. Waasdorp J a, Evian Cl, Mandracchia M. Immediate Placement of Implants Into Infected Sites: A Systematic Review of the Literature. J Periodontol. 2010;81(6):801-8.

8. Blus C, Szmukler-Moncler S, Khoury P, Orrù G. Immediate Implants Placed in Infected and Noninfected Sites after Atraumatic Tooth Extraction and Placement with Ultrasonic Bone Surgery. Clin Implant Dent Relat Res. 2015;17(1):287-97.

9. Mello CC, Lemos CAA, Verri FR, Santos DM, Goiato MC. Immediate implant placement into fresh extraction sockets versus delayed implants into healed sockets : A systematic review and meta-analysis. Int J Oral Maxillofac Surg. International Association of Oral and Maxillofacial Surgery; 2017;03:01-16.

10. Bersani E, Ricciardi Coppede A, Pinto Prata HH de P. Immediate Loading of Implants Placed in Fresh Extraction Sockets in the Molar Area with Flapless and Graftless Procedures : A Case Series. Int J Periodontics Restorative Dent. 2010;30:291-9.

11. Ramalingam S, Al-Hindi M, Al-Eid RA, Nooh N. Clinical evaluation of implant survival based on size and site of placement: A retrospective study of immediate implants at single rooted teeth sites. Saudi Dent J. King Saud University; 2015;27(2):105-11.

12. Block MS. Placement of Implants Into Fresh Molar Sites : Results of 35 Cases. J Oral MaxiIlofac Surg. Elsevier Inc.; 2011;69(1):170-4.

13. Jensen OT. Dental extraction, Immediate placement of Dental implants, and Immediate Function. Oral Maxillofac Surg Clin N Am. Elsevier Inc; 2015;27(2):273-82.

14. Altintas NY, Taskesen F, Bagis B, Baltacioglu E, Cezairli B, Senel FC. Immediate implant placement in fresh sockets versus implant placement in healed bone for full-arch fixed prostheses with conventional loading. Int J Oral Maxillofac Surg. International Association of Oral and Maxillofacial Surgery; 2015;1-6.

15. Hu C, Gong T, Lin W, Yuan Q, Man Y. Immediate implant placement into posterior sockets with or without buccal bone dehiscence defects : A retrospective cohort study. J Dent. Elsevier; 2017;1-6.

16. Lin Z, Fateh A, Salem DM, Intini G. Periosteum : Biology and Applications in Craniofacial Bone Regeneration. J Dent Res. 2013;1-8.

17. Schwartz-Arad D, Chaushu G. Placement of implants into fresh extraction sites: 4 to 7 years retrospective evaluation of 95 immediate implants. J Periodontol. 1997;68(11):11106. 
18. Pluemsakunthai W, Le B, Kasugai S. Effect of Buccal Gap Distance on Alveolar Ridge Alteration After Immediate Implant Tomographic and Morphometric analysis in dogs. Implant Dent. 2015;24(1):70-6.

19. Fugazzotto PA. Implant Placement at the Time of Maxillary Molar Extraction: Technique and Report of Preliminary Results of 83 Sites. J Periodontol. 2006;77(2):302-9.

20. Barone A, Cornelini R, Ciaglia R, Covani U. Implant Placement in Fresh Extraction Sockets and Simultaneous Osteotome Sinus Floor Elevation: A Case Series. Int J Periodontics Restorative Dent. 2008;28:283-9.

21. Cornelini R, Cangini DDSF, Covani U, Thomas DDS, Jr GW. Immediate Restoration of Implants Placed into Fresh Extraction Sockets for Single-Tooth Replacement : A Prospective Clinical Study. Int J Periodontics Restorative Dent. 2005;25:439-47.

22. Bell CL, Diehl D, Bell BM, Bell RE. The immediate placement of dental implants into extraction sites with periapical lesions: A retrospective chart review. J Oral Maxillofac Surg. Elsevier Inc.; 2011;69(6):1623-7.

23. Cochran DL, Nummikoski P V, Schoolfield JD, Jones AA, Oates TW. A Prospective Multicenter 5-Year Radiographic Evaluation of Crestal Bone Levels Over Time in 596 Denta Implants Placed in 192 Patients. J Periodontol. 2009;80(5):725-33. 